\title{
纳米颗粒对 $\mathrm{Ni}$ 基复合镀渗层耐冲蚀性能的影响
}

\author{
徐 江 ${ }^{1, *}$ 卓城之 ${ }^{1}$ 蒋书运 ${ }^{2}$ \\ (' 南京航空航天大学材料科学与技术学院, 南京 210016; 2 东南大学机械工程学院, 南京 210096)
}

\begin{abstract}
摘要：采用复合镀渗工艺在 $316 \mathrm{~L}$ 不锈钢表面分别制备了两种纳米颗粒(非晶 nano- $\mathrm{SiO}_{2}$ 颗粒和 nano-SiC 颗粒) 增强的 $\mathrm{Ni}$ 基复合镀渗合金层. 利用扫描电子显微镜 $(\mathrm{SEM})$ 和透射电子显微镜(TEM)观察了两种复合镀渗合金层 的微观组织特征. 通过外加电位 $(+0.2 \mathrm{~V})$ 条件下的电流密度、冲蚀条件下的极化曲线和冲蚀后的交流阻抗谱表征 了这两种复合镀渗层在单相流 $(3.5 \%$ ( $w$, 质量分数 $) \mathrm{NaCl}$ 溶液)与料浆流( $3.5 \% \mathrm{NaCl}+10 \%$ ( $w$ )石英砂)中的电化学腐 蚀特征, 并采用 SEM 观察两种复合镀渗层冲蚀后的截面形貌, 探讨这两种纳米颗粒增强的 Ni 基复合镀渗层在 料浆流中的冲蚀机理. 电化学测试结果表明: 静态条件下, 电刷镀含纳米 $\mathrm{SiO}_{2}$ 颗粒的复合镀渗层的耐蚀性能低 于单一合金层, 而动态冲蚀条件下, 结果与之相反; 电刷镀含 $\mathrm{SiC}$ 颗粒的复合镀渗层的而蚀性能在静态和动态冲 蚀条件下均低于单一合金层. 对两种复合镀渗层的冲蚀截面形貌观察表明: 弥散分布的纳米 $\mathrm{SiO}_{2}$ 颗粒能明显改 善 $\mathrm{Ni}$ 基合金层的耐冲蚀性能; 而添加纳米 $\mathrm{SiC}$ 颗粒在渗金属过程中已完全分解, 导致三元硅化物 $\left(\mathrm{Cr}_{6.5} \mathrm{Ni}_{2.5} \mathrm{Si}\right)$ 和 碳化物 $\left(\mathrm{Cr}_{23} \mathrm{C}_{6}\right)$ 的析出, 而析出相在冲蚀过程易于脱落, 加速了 $\mathrm{Ni}$ 基合金层的质量流失.
\end{abstract}

关键词：纳米颗粒；复合镀渗；极化曲线；磨蚀；交流阻抗谱 中图分类号： O646; TG172.5

\section{Effect of Nanoparticles on the Erosion-Corrosion Resistance Property of a Ni-Based Alloying Layer}

\author{
XU Jiang ${ }^{1, *} \quad$ ZHUO Cheng-Zhi ${ }^{1} \quad$ JIANG Shu-Yun ${ }^{2}$ \\ $\left({ }^{1}\right.$ College of Material Science and Technology, Nanjing University of Aeronautics and Astronautics, Nanjing 210016, P. R. China; \\ ${ }^{2}$ School of Mechanical Engineering, Southeast University, Nanjing 210096, P. R. China)
}

\begin{abstract}
A nanoparticle reinforced Ni-based alloying layer was prepared by a duplex surface treatment on the surface of AISI 316L stainless steel. This steel contained $\mathrm{Ni} /$ nano- $\mathrm{SiO}_{2}$ or $\mathrm{Ni} /$ nano-SiC layer which was predeposited by brush plating and subsequent surface alloying with $\mathrm{Ni}-\mathrm{Cr}-\mathrm{Mo}-\mathrm{Cu}$ by a double glow process. The microstructures of the two kinds of nanoparticles that reinforced the Ni-based alloying layers were investigated by scanning electron microscopy (SEM) and transmission electron microscopy (TEM). The corrosion behaviors of the composite layers under hydrodynamic conditions and at different rotational speeds were characterized by current responses at a potential of +0.2 $\mathrm{V}$, a potentiodynamic polarization curve and electrochemical impedance spectroscopy (EIS) under a static state (3.5\% ( $w$, mass fraction) $\mathrm{NaCl}$ solution) and under slurry flow conditions $(3.5 \%(w) \mathrm{NaCl}$ solution $+10 \%(w)$ sand particles). To assess possible erosion-corrosion mechanisms, the worn sample surfaces were observed by SEM. Electrochemical tests showed that the corrosion resistance of the composite layer with the brush plated $\mathrm{Ni} /$ nano-SiO $\mathrm{O}_{2}$ particle interlayer was slightly lower than that of the single Ni-based alloying layer produced under static state conditions. However, under hydrodynamic conditions, the corrosion resistance of the composite layer with the brush plated Ni/nano- $\mathrm{SiO}_{2}$ particle interlayer was obviously superior to that of the single Ni-based alloying layer. The corrosion resistance of the composite layer produced with the brush plated $\mathrm{Ni} /$ nano-SiC particle interlayer was lower than that of the single Ni-
\end{abstract}

Received: March 6, 2009; Revised: June 1, 2009; Published on Web: July 31, 2009.

*Corresponding author. Email: xujiang73@nuaa.edu.cn; Tel: +86-25-84236257.

The project was supported by the National Natural Science Foundation of China (50704022) and Natural Science Foundation of Jiangsu Province, China (BK2007591).

国家自然科学基金(50704022)和江苏省自然科学基金(BK2007591)资助项目

C Editorial office of Acta Physico-Chimica Sinica 
based alloying layer produced under static state and hydrodynamic conditions. From the eroded-corroded cross-section morphologies we found that highly dispersive nano- $\mathrm{SiO}_{2}$ particles were helpful in improving the erosion-corrosion resistance of the Ni-based alloying layer whereas the carbides and silicide phases were deleterious to the Ni-based alloying layer.

Key Words: Nanoparticle; Duplex surface treatment; Polarization curve; Erosion-corrosion;

Eelectrochemical impedance spectroscopy

冲刷腐蚀(冲蚀)现象广泛存在于机械、石油化 工、冶金采矿和能源交通等各个领域 ${ }^{[1-3]}$. 由于在冲 蚀过程中, 腐蚀和冲刷之间存在较大的交互作用 ${ }^{[4,5]}$, 因此在发展耐冲蚀合金时要综合考虑材料的耐蚀性 能和耐磨性能. 而蚀合金设计通常考虑通过添加合 金元素增强其自身针化能力, 往往不希望基体中存 在易导致形成微观腐蚀原电池的第二相; 而耐磨合 金设计采用高硬度的第二相强化以提高合金的承受 载荷和抗犁削能力. 寻求耐蚀性和耐磨性的最佳搭 配使两者之间的交互作用最小, 有可能是发展耐冲 蚀材料的最佳途径. 合金中加人硬质第二相是提高 材料耐磨性能有效手段之一, 但硬质第二相的加人 对金属基复合材料的耐蚀性能和耐冲蚀性能的影响 较为复杂, 国内外学者的相关研究结果并不一致 ${ }^{[6-12]}$. Szczygieł 等人同对比研究了 $\mathrm{Ni} / n-\mathrm{Al}_{2} \mathrm{O}_{3}$ 复合镀层与 $\mathrm{Ni}$ 镀层的耐蚀性能, 认为弥散分布于镀层中具有绝 缘特性的纳米 $\mathrm{Al}_{2} \mathrm{O}_{3}$ 颗粒的 “屏蔽作用” 以及钝化膜 中低密度的缺陷(孔洞和裂纹)是其耐蚀性能优于 $\mathrm{Ni}$ 镀层的主要原因; 相反, Pardo 等人研究了 $\mathrm{SiC}$ 颗 粒增强 AZ91 镁合金分别在三种条件下 $(3.5 \%(w$, 质 量分数) $\mathrm{NaCl}$ 溶液, 中性和相对较高湿度环境中的 盐雾试验)的腐蚀性能, 结果发现: 添加的 $\mathrm{SiC}$ 颗粒 增加了合金的腐蚀速率; 而且随着暴露时间的延长, 促进腐蚀产物的剥落. 第二相增强复合材料的冲蚀 性能影响因素相比静态腐蚀条件下更为复杂, 它不 仅与腐蚀介质流速、悬浮于腐蚀介质中固体颗粒的 硬度和含量有关, 也与材料中位错密度、增强相电化 学稳定性、增强相与基体合金间界面相有关. $\mathrm{Tu}$ 等 人 ${ }^{[100}$ 研究了 $19.5 \%$ ( $\varphi$, 体积分数 $)$ 的 $\mathrm{Al}_{18} \mathrm{~B}_{4} \mathrm{O}_{33}$ 晶须增 强 AC4C Al 合金在料浆中的耐冲蚀性能. 研究结果 表明: 对比 $\mathrm{AC} 4 \mathrm{C} \mathrm{Al}$ 合金, 在低速条件下, 由于添加 了 $\mathrm{Al}_{18} \mathrm{~B}_{4} \mathrm{O}_{33}$ 晶须提高了复合材料的硬度, 进而改善 其耐冲蚀性能; 而在高速条件下, 添加 $\mathrm{Al}_{18} \mathrm{~B}_{4} \mathrm{O}_{33}$ 晶 须不利于铝基复合材料的耐冲蚀性能的提高. Das 等人 ${ }^{[11]}$ 研究含 $10 \%-15 \%(w)$ 的 $\mathrm{SiC}$ 增强 LM13 铝-硅 合金在含 $0-40 \%(w)$ 石英砂人造海水和模拟采矿腐
蚀介质条件中的耐冲蚀性能, 其结果表明, 在两种腐 蚀介质条件下, $\mathrm{SiC}$ 增强 LM13 合金的冲蚀失重率 明显小于不含碳化物增强的 LM13 铝-硅合金. $\mathrm{SiC}$ 颗粒对铝-硅合金的影响具有两面性:一方面, $\mathrm{SiC}$ 是绝缘体, 不易受到腐蚀, 减少暴露于腐蚀溶液中金 属基体的面积,有利于耐蚀性能的提高; 另一方面其 存在又破坏了钝化膜的连续性, 对耐蚀性能不利.

在冲蚀过程中, 金属材料表层钝化膜的化学性 质及机械性质对其耐冲蚀性能必然会产生影响, 而 传统的冲蚀失重法难以评价钝化膜在冲蚀过程中的 修复过程及破坏规律. 目前, 国内外仅有少量报道 ${ }^{[13-16]}$ 通过动态过程中的电流密度测试来表征针化膜的行 为; 采用交流阻抗谱来评价冲蚀介质对金属表面钝 化膜的 “损伤程度” 的方法也仅有少量报道 ${ }^{[17,18]}$. 因 此, 本文通过外加电位 $(+0.2 \mathrm{~V})$ 条件下的电流密度、 动态冲蚀条件下的极化曲线和冲蚀后的交流阻抗 谱表征两种纳米颗粒增强的 $\mathrm{Ni}$ 基复合镀渗合金层 在单相流 $(3.5 \%(w) \mathrm{NaCl}$ 溶液)与料浆流 $(3.5 \%(w)$ $\mathrm{NaCl}+10 \%(w)$ 石英砂)中的电化学腐蚀特征, 并研究 钝化膜的修复过程及稳定性, 探讨 $\mathrm{Ni}$ 基复合镀渗层 中的不同颗粒对其耐冲蚀性能影响规律.

\section{1 实验部分}

\section{1 实验材料与制备方法}

试样材料为市售轧制 $316 \mathrm{~L}$ 不锈钢, 其成分为 $(w(\%))$ : C 0.021, Cr 17.61, Ni 12.45, Mo 2.29, Si 0.69, $\mathrm{Mn} 1.05, \mathrm{~S} 0.020, \mathrm{P} 0.031, \mathrm{Fe}$ 余量. 试样尺寸为 60 $\mathrm{mm} \times 30 \mathrm{~mm} \times 4 \mathrm{~mm}$. 纳米陶瓷颗粒增强 $\mathrm{Ni}$ 基镀渗合 金层的制备由两个过程组成: 首先是在 $316 \mathrm{~L}$ 不锈 钢基体上利用电刷镀技术得到两种厚度为 20-30 $\mu \mathrm{m}$ 的非晶态 $n-\mathrm{SiO}_{2}$ 和 $n-\mathrm{SiC}$ 增强的复合电刷镀镀 层 (复合镀液为每升快速镍镀液中加人 $20 \mathrm{~g}$ 、颗粒 尺寸为 20-30 nm 的非晶态 $\mathrm{SiO}_{2}$ 或 $\mathrm{SiC}$, 工艺流程 见文献 [19]), 纳米颗粒在刷镀层中的质量分数约为 6\%-7\%. 随后在自制双层辉光离子渗金属炉中进行 $\mathrm{Ni}-\mathrm{Cr}-\mathrm{Mo}-\mathrm{Cu}$ 多元共渗. 采用脉冲放电模式: 源极采 
用直流电源, 工件采用脉冲电源. 源极材料为Hastelloy C-2000 合金, 成分为 $(w(\%)): \mathrm{Ni} 59, \mathrm{Mo} 16, \mathrm{Cr} 23, \mathrm{Cu}$ $1.6, \mathrm{C}<0.01$, 源极尺寸为 $130 \mathrm{~mm} \times 50 \mathrm{~mm} \times 4 \mathrm{~mm}$. 渗 金属工艺参数见文献[19]. 为了叙述简洁, 文中定义 以预沉积非晶态 $\mathrm{Ni} / \mathrm{nano}^{-} \mathrm{SiO}_{2}$ 复合刷镀层+双辉多 元共渗工艺所制备的复合镀渗层为复合层 $\mathrm{A}$, 以预 沉积 Ni/nano-SiC 的复合刷镀层+双辉多元共渗工 艺所制备的复合镀渗层为复合层 $\mathrm{B}$, 在 $316 \mathrm{~L}$ 不锈钢 表面直接进行 $\mathrm{Ni}-\mathrm{Cr}-\mathrm{Mo}-\mathrm{Cu}$ 多元共渗所得到的合 金渗层为单一合金层.

\section{2 分析方法}

采用 Quanta200 型扫描电子显微镜对表面层横 截面组织与腐蚀形貌进行了观察. 利用线切割从试 样表面切下 $0.2 \mathrm{~mm}$ 薄片, 由靠基体侧用砂纸机械 研磨减薄至 $50 \mu \mathrm{m}$ 左右, 然后采用双喷电解法制成 薄膜样品, 在 Tecnai G220 透射电镜下观察复合镀 渗层中第二相形貌及精细结构. 采用自制的料浆式 冲蚀实验评价纳米颗粒增强复合镀渗层以及对比材 料的而冲蚀性能. 该冲蚀试验机 ${ }^{[20]}$ 是在参考文献[21] 基础之上自行设计, 主要由三部分组成: 速度调节系 统、电化学测试系统和料浆槽装置系统. 其中, 速度 调节系统与电机 $\left(0-4000 \mathrm{r} \cdot \mathrm{min}^{-1}\right.$ 无级可调, 误差 \pm 3 $\mathrm{r} \cdot \mathrm{min}^{-1}$ )连接. 实验过程中, 实样的线速度分别为 1.88 、 $2.51 、 2.98$ 和 $3.45 \mathrm{~m} \cdot \mathrm{s}^{-1}$; 电化学测试系统与 $\mathrm{CHI} 660 \mathrm{~b}$ 连接, 电化学实验选取三电极体系, 以饱和甘录电极 (SCE) 作参比电极, Pt 片作为辅助电极. 动态条件下 的电流(外加电位 $+0.2 \mathrm{~V}$ )、极化曲线和交流阻抗测试 (EIS)均采用 CHI660c 型电化学测试系统, 在敞开的 大气环境中进行. 极化曲线测试开始前, 先浸泡或冲 蚀 $30 \mathrm{~min}$ 以得到稳定的开路电位(OCP)后开始测 量, 扫描速率为 $30 \mathrm{mV} \cdot \mathrm{min}^{-1}$. 交流阻抗(EIS)测试 选区交流正弦激励信号, 幅值为 $5 \mathrm{mV}$, 测试频率范 围为 $10^{-2}-10^{5} \mathrm{~Hz}$. 阻抗的测试条件为: 几种测试材料 分别经过浸泡 $20 \mathrm{~h}$ 、单相流( $3.5 \% \mathrm{NaCl}$ 溶液)和料浆 流 $(3.5 \% \mathrm{NaCl}+10 \%$ 石英砂) 冲蚀 $20 \mathrm{~h}$. 电化学试样非 工作面均用环氧树脂密封, 试样工作面 $(10 \mathrm{~mm} \times 10$ $\mathrm{mm}$ ) 预磨至 800 \# 砂纸, 用丙酮超声清洗, 干燥后待 电化学测试使用. 实验温度为 $(20 \pm 1){ }^{\circ} \mathrm{C}$. 试样安装在 旋转圆盘轮的燕尾槽上, 槽的底部装有铜柱、弹簧和 引线, 连同碳刷-铜环一起实现研究电极电化学试验 信号的导出. 实验中使用的石英砂粒度为 60-80 目. 冲蚀实验完成后采用 SEM 观察冲蚀形貌.

\section{2 实验结果与讨论}

\section{1 微观组织分析}

图 1 为在 316L 不锈钢表面所形成的两种复合 合金层的截面 SEM 照片、合金元素沿深度分布变化 曲线以及微观组织 TEM 形貌照片. 由图 $1(a, b)$ 可见, 两种表面合金层的组织均匀、连续、致密, 没有孔洞、 裂纹等缺陷存在, 复合层的厚度约为 $65 \mu \mathrm{m}$. 成分 分布沿深度变化曲线(图 l(c, d))表明: 形成两种复 合镀层的表面合金成分与源极材料的合金成分相接 近; 预先刷镀的试样在双辉多元合金共渗的高温条 件下 $\left(1000{ }^{\circ} \mathrm{C}\right)$, 发生了两个界面合金元素的扩散, 即 由源极溅射沉积到刷镀层表面的合金元素向内扩 散, 以及刷镀层与不锈钢基体界面之间的互扩散. 扩 散造成的“稀释作用”导致复合镀渗层的硬度相比 刷镀层有明显降低, 但仍明显高于单一合金层(见表 1). 由图 1(e)可见: 复合层 A 中的球状颗粒弥散分布 于 $\mathrm{Ni}$ 基固溶体中, 颗粒与基体之间无界面相生成, 且颗粒的尺寸与添加到镀液中的颗粒尺寸相当. 经 选区电子衍射花样标定结果表明, 添加到刷镀层中 的非晶纳米 $\mathrm{SiO}_{2}$ 颗粒, 在高温处理后仍保持非晶态. 图 1(f)为复合层 B 的微观组织 TEM 形貌. 经选区电 子衍射花样标定结果表明, 多元共渗的高温作用下, 原刷镀层中的 $\mathrm{SiC}$ 颗粒已完全分解, 并与基体中的 合金元素 $\mathrm{Ni}$ 和 $\mathrm{Cr}$ 反应, 沿晶界生成棒状碳化物 $\mathrm{Cr}_{23} \mathrm{C}_{6}$ 和在晶内生成三元硅化物 $\mathrm{Cr}_{6.5} \mathrm{Ni}_{2.5} \mathrm{Si}$.

\section{2 冲蚀条件下的电流密度与开路电位测量}

试样旋转速度随时间的变化曲线如图 2(A)所示. 图 2(B, C) 是复合层 $\mathrm{A}$ 和复合层 $\mathrm{B}$ 、单一合金层以及 $316 \mathrm{~L}$ 不锈钢基材在单相流( $3.5 \% \mathrm{NaCl}$ 溶液)和料浆流 $(3.5 \% \mathrm{NaCl}+10 \%$ 石英砂 $)$ 介质中, 外加电位 $+0.2 \mathrm{~V}$, 不 同旋转速度条件下, 电流密度随时间变化曲线. 由图 2 (B) 可见, 随旋转速度的增加, 复合层 $\mathrm{B}$ 和 $316 \mathrm{~L}$ 不 锈钢均出现明显波动, 而复合层 $\mathrm{A}$ 和单一合金层的 电流密度较稳定. 静态条件下, $316 \mathrm{~L}$ 不锈钢的电流 密度较稳定, 基本维持在 $10^{-6} \mathrm{~A} \cdot \mathrm{cm}^{-2}$ 数量级. 实验 数据显示: 当试样的旋转速度增至 $1.88 \mathrm{~m} \cdot \mathrm{s}^{-1}$ 时, 流 经 $316 \mathrm{~L}$ 不锈钢的电流密度迅速增加; 当试样旋转 速度增至 2.51 和 $2.98 \mathrm{~m} \cdot \mathrm{s}^{-1}$ 时, $316 \mathrm{~L}$ 不锈钢的电流 密度整体上又趋于稳定, 维持在 $10^{-4} \mathrm{~A} \cdot \mathrm{cm}^{-2}$ 数量级; 当速度增加到 $3.45 \mathrm{~m} \cdot \mathrm{s}^{-1}$ 时, 电流密度进一步增加, 直至测试试验结束, 仍然没有达到稳定值. 复合层 $\mathrm{B}$ 的电流密度在静态条件下有轻微的波动; 当旋转速 度增加到 $1.88 \mathrm{~m} \cdot \mathrm{s}^{-1}$ 时, 电流密度逐渐增加, 电流密 

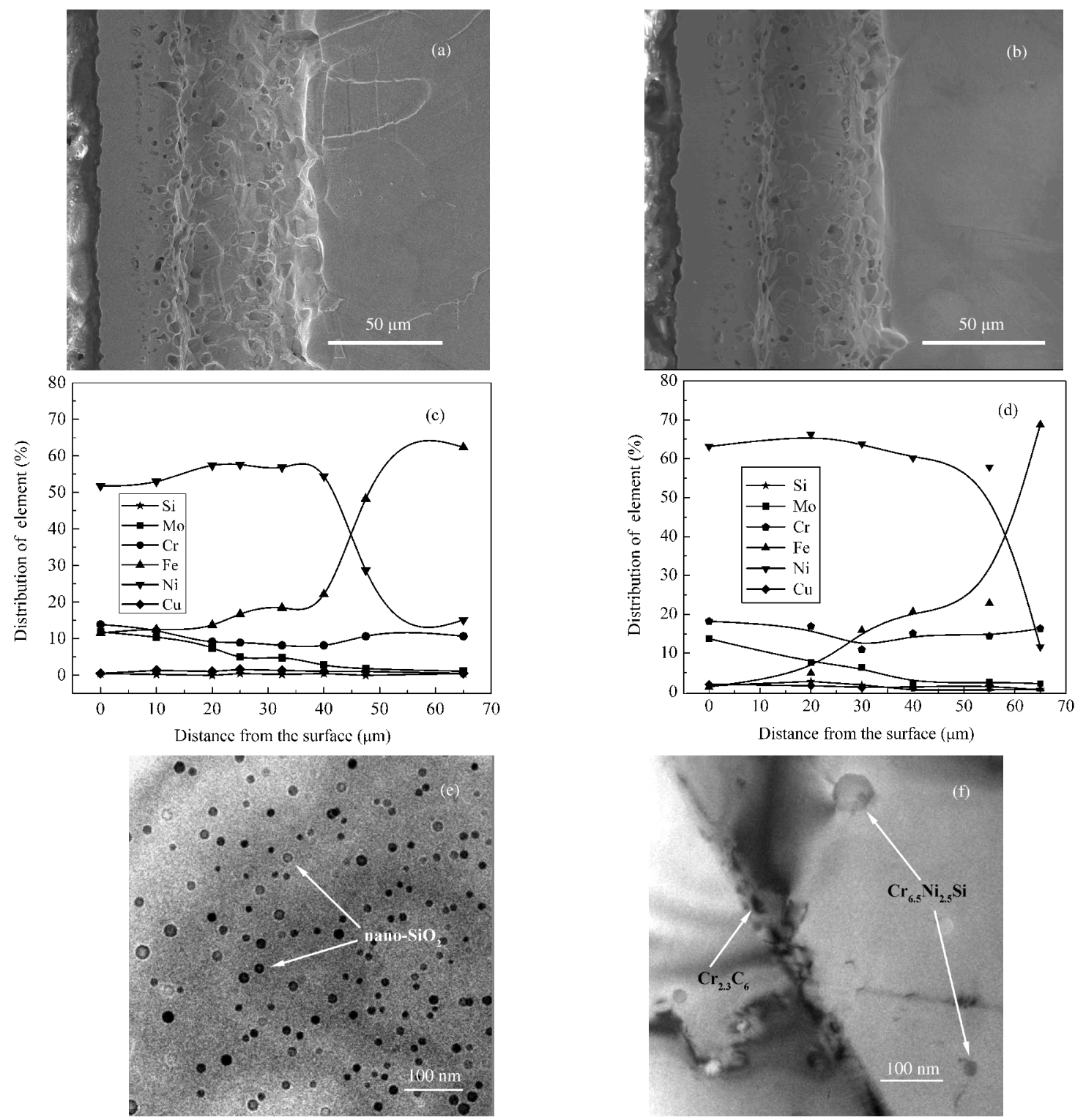

图 1 复合镀渗层 A (a, c,e)和 B (b, d, f) 的截面 SEM 照片 $(a, b)$, 成分 $(w)$ 沿深度分布变化曲线 $(c, d)$ 及 TEM 照片 $(e, f)$

Fig.1 Cross-section SEM images $(a, b)$, chemical composition distribution $(w)$ change curves along the deepness $(c, d)$, and TEM images (e, f) of composite alloying layers $A(a, c, e)$ and $B(b, d, f)$

Composite alloying layer with brush plating $\mathrm{Ni} / \mathrm{nano}-\mathrm{SiO}_{2}$ particles interlayer and composite alloying layer with brush plating $\mathrm{Ni} / \mathrm{nano}-\mathrm{SiC}$ particles interlayer were designated as composite alloying layer A and composite alloying layer $\mathrm{B}$, respectively.

度曲线呈现一定程度的粗化; 当旋转速度分别增至 2.51 和 $2.98 \mathrm{~m} \cdot \mathrm{s}^{-1}$ 时, 电流密度曲线出现显著的波 动; 当旋转速度达到 $3.45 \mathrm{~m} \cdot \mathrm{s}^{-1}$ 时, 电流密度逐渐增 大, 曲线粗化愈加明显. 对比复合层 $\mathrm{A}$ 和单一合金 层的电流密度-时间曲线表明: 两种材料在测试的 旋转速度范围内, 电流密度明显小于前两者. 当速度
低于 $1.88 \mathrm{~m} \cdot \mathrm{s}^{-1}$ 时, 两种材料的电流密度都较稳定; 当速度增加到 $2.51 \mathrm{~m} \cdot \mathrm{s}^{-1}$ 时, 单一合金层的电流密 度曲线出现了明显的波动, 而复合层 $\mathrm{A}$ 仍然保持稳 定的电流密度; 随着旋转速度进一步的增加, 单一 合金层的电流密度明显大于复合层 $\mathrm{A}$. 当介质中加 人 $10 \%(w)$ 的石英砂后, 随着旋转速度的增加, 几种

表 1 几种表面合金层与 $316 \mathrm{~L}$ 不锈钢的硬度

Table 1 Hardness of the surface alloying layers and 316L stainless steel

\begin{tabular}{|c|c|c|c|c|c|c|}
\hline Sample & $\begin{array}{c}\text { 316L stainless } \\
\text { steel }\end{array}$ & $\begin{array}{c}\text { composite alloying } \\
\text { layer A }\end{array}$ & $\begin{array}{c}\text { composite alloying } \\
\text { layer B }\end{array}$ & $\begin{array}{c}\text { singl alloying } \\
\text { layer }\end{array}$ & $\begin{array}{c}\mathrm{Ni} / \text { nano- } \mathrm{SiO}_{2} \text { brush } \\
\text { plating layer }\end{array}$ & $\begin{array}{c}\mathrm{Ni} / \text { nano-SiC brush } \\
\text { plating layer }\end{array}$ \\
\hline $\begin{array}{l}\text { Hardness } \\
\left(\mathrm{HV}_{0.1}\right)\end{array}$ & 235 & 368 & 311 & 202 & 480 & 512 \\
\hline
\end{tabular}



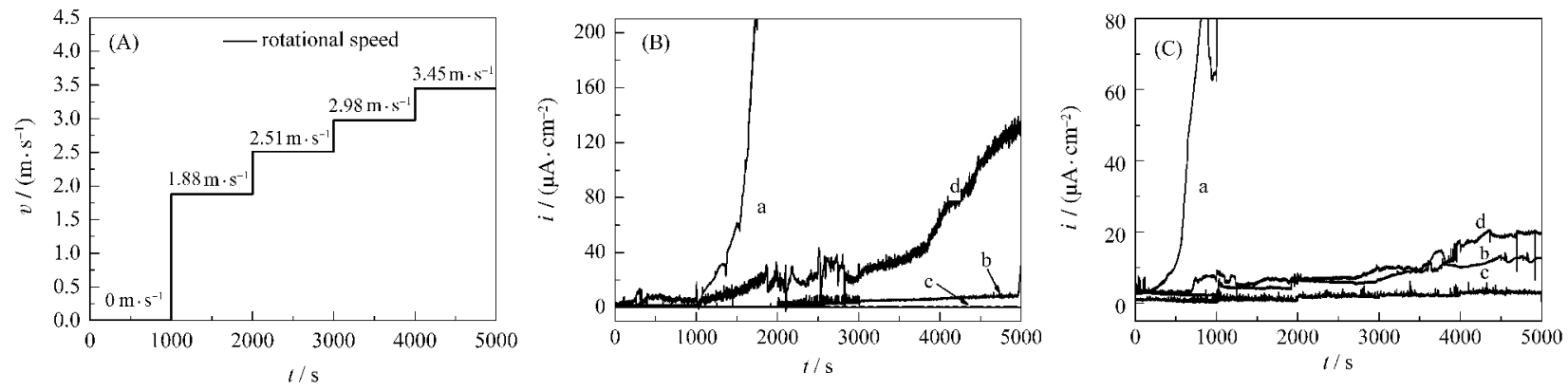

图 2 (A)冲蚀过程中试样的旋转速度与测试时间的关系曲线图及 $(B, C)$ 试样在不同条件下电流密度随测试时间的变化曲线

Fig.2 (A) Relationship of the rotational speed and measured time of samples in the erosion process,

$(B, C)$ current density responses of samples $v$ s time at different conditions

In Fig.3(B, C), 316L stainless steel (a), single Ni-based alloying layer (b), composite alloying layer A (c) and layer B (d) measured at a potential of $+0.2 \mathrm{~V}$ in liquid flow $(\mathrm{B})$ and slurry flow $(\mathrm{C})$ as a function of measured time, and the measured time corresponding to the rotational speed is in accordance with that in Fig.2(A).

测试材料的电流密度都相应增加, 其中尤以 $316 \mathrm{~L}$ 不锈钢的电流密度增加最为明显, 如图 2(C) 所示. 在较高旋转速度条件下, 复合层 $\mathrm{B}$ 、单一合金层和 $316 \mathrm{~L}$ 不锈钢的电流密度曲线均出现了明显的波动, 而复合层 $\mathrm{A}$ 的电流密度曲线相对较平稳.

Burstein 等人 ${ }^{[13]}$ 通过电化学噪声法和电流密度 随时间变化曲线研究了 304 不锈钢在料浆流冲蚀过 程中, 固相颗粒的冲击对电流密度的影响. 认为电流 密度的突然增大是由于试样表面的去钝化过程导致 的, 即当固相颗粒冲击试样表面时, 钝化膜被去除, 新鲜基体暴露于腐蚀介质中, 从而使得电流密度增 大. 钝化膜的持续剥落与形成导致电流密度的连续 波动. 因此, 由图 2(C) 可知, 在静态或低旋转速度条 件下, 固相颗粒对测试材料表面氧化膜的去除作用 弱, 材料表面存在较完整的钝化膜, 电流密度波动较 小; 而高旋转速度下, 固相颗粒的冲击能量高, 材料

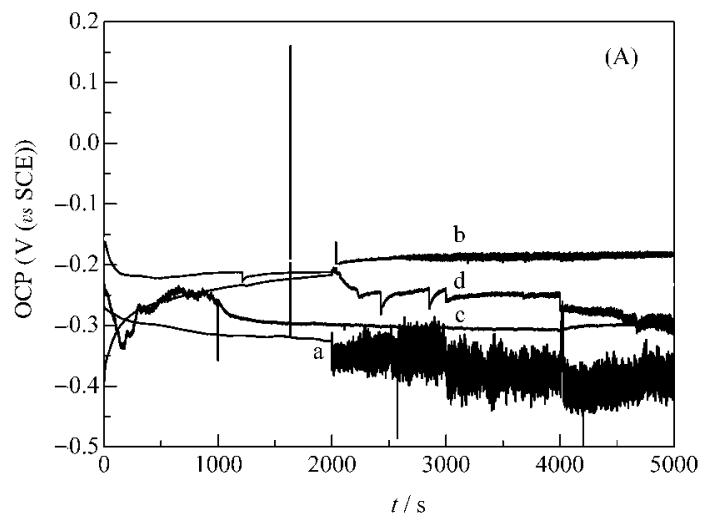

表面所受剪切力和冲击力增大, 使得材料表面钝化 膜易破裂, 电流密度增加. 316L 不锈钢的波动范围 大, 证实其钝化膜的修复作用小于硬质颗粒的机械 去除作用, 针化膜的修复能力最弱; 复合层 B 的电 流密度波动出现在更高转速条件下, 说明其针化膜 的修复能力优于 $316 \mathrm{~L}$ 不锈钢, 但明显低于复合层 $\mathrm{A}$ 和单一合金层. 复合层 $\mathrm{A}$ 在整个测试周期内, 电 流密度均保持平稳, 证明其钝化膜的修复能力最佳.

图 3(A) 和 3(B) 分别是复合层 $\mathrm{A}$ 、复合层 $\mathrm{B}$ 和两 种对比材料(单一合金层和 $316 \mathrm{~L}$ 不锈钢) 在不同旋 转速度 $\left(0 、 1.88 、 2.51 、 2.98\right.$ 和 $\left.3.45 \mathrm{~m} \cdot \mathrm{s}^{-1}\right)$ 条件下, 在 单相流 (3.5\% NaCl 溶液) 和料浆流 $(3.5 \% \mathrm{NaCl}+10 \%$ 石英砂)介质中, 开路电位 $(\mathrm{OCP})$ 与时间的关系图(测 试时间与旋转速度的关系与图 2(A)一致). 由图 3(A) 可见, 在单相流条件下, $316 \mathrm{~L}$ 不锈钢在静态和低旋 转速度 $\left(1.88 \mathrm{~m} \cdot \mathrm{s}^{-1}\right)$ 的冲蚀条件下, OCP 比较稳定;

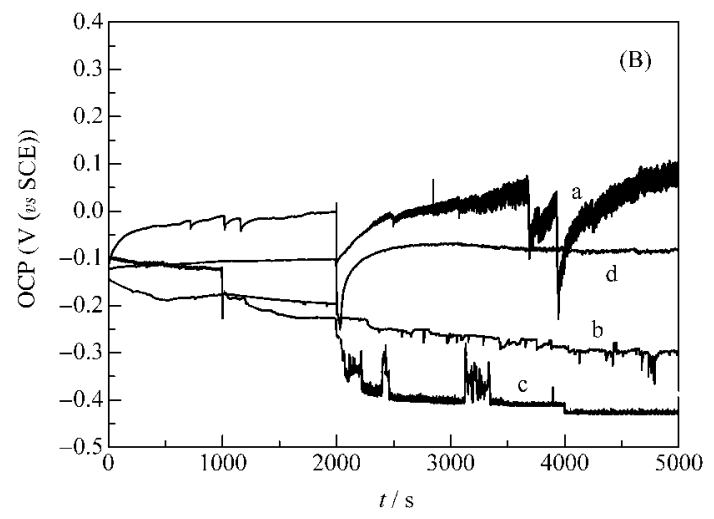

图 3 试样在单相流(A)和料浆流(B)介质中不同旋转速度条件下开路电位 $(\mathbf{O C P})$ 随测试时间的变化曲线

Fig.3 Open circuit potential (OCP) as a function of measured time for samples measured in liquid flow (A) and in slurry flow (B)

(a) 316L stainless steel, (b) single Ni-based alloying layer, (c) composite alloying layer A, (d) composite alloying layer B; In Fig.3, the measured time corresponding to the rotational speed is in accordance with that in Fig.2(A). 
而当旋转速度大于 $1.88 \mathrm{~m} \cdot \mathrm{s}^{-1}$ 时, OCP 曲线出现了 较大的连续波动, $\mathrm{OCP}$ 曲线严重粗化. Ambat 等人 ${ }^{[22]}$ 研究了 $\mathrm{Al}-\mathrm{Fe}$ 合金在 $3.5 \%(w) \mathrm{NaCl}$ 溶液中的 $\mathrm{OCP}$ 随时间的变化曲线, 认为开路电位出现较大的连续 波动(表现为 $\mathrm{OCP}-t$ 曲线的粗化)是材料表面发生的 点蚀所引起. 因此, 当旋转速度大于 $1.88 \mathrm{~m} \cdot \mathrm{s}^{-1}$ 时, $316 \mathrm{~L}$ 不锈钢表面已经发生点蚀. 对于单一合金层和 复合层, 在静态和低速冲蚀条件下, OCP 相对比较 稳定, 没有大的连续波动出现, 说明在低旋转速度条 件下, 速度的变化对材料表面状态影响较小, 材料表 面相对稳定. 在高旋转速度条件下 $\left(3.45 \mathrm{~m} \cdot \mathrm{s}^{-1}\right)$, 仅有 复合层 $\mathrm{B}$ 出现了连续波动, 表明复合层 $\mathrm{B}$ 表面已开 始发生点蚀. 由图 3(B) 可见, 在料浆流条件下, 在静 态和低旋转速度 $\left(1.88 \mathrm{~m} \cdot \mathrm{s}^{-1}\right)$ 冲蚀条件下, 几种测试材 料的 $\mathrm{OCP}$ 较为稳定; 当旋转速度大于 $1.88 \mathrm{~m} \cdot \mathrm{s}^{-1}$ 时, 复合层 $\mathrm{A}$ 和单一合金层的 $\mathrm{OCP}$ 曲线波动较小, 表 明这两种材料具有较强的钝化膜修复能力; 而复合 层 $\mathrm{B}$ 和 $316 \mathrm{~L}$ 不锈钢则出现较大的波动, 证实这两 种材料钝化膜的修复能力较差, 较高的旋转速度下
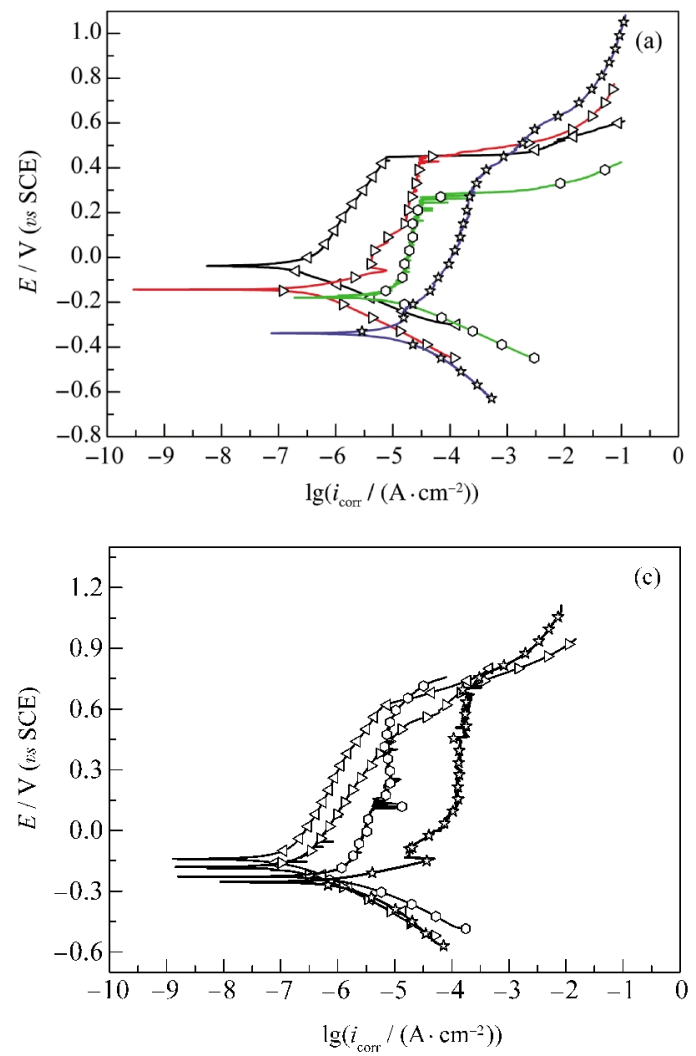

易于发生点蚀.

\section{3 极化曲线测试}

图 4 为四种测试试样在不同旋转速度冲蚀条件 下的极化曲线. 由图可见, 几种材料的腐蚀电流密度 $\left(i_{\text {corr }}\right)$ 都随着旋转速度的增加而增大. $i_{\text {corr }}$ 越大, 材料 的腐蚀速率就越大, 即耐蚀性能就越差. 因此旋转速 度增大, 测试材料的耐蚀性能均降低. 单一合金层和 复合层 $\mathrm{A}$ 在静态和动态冲蚀条件下, 均有明显钝化 现象; 复合层 $\mathrm{B}$ 和 $316 \mathrm{~L}$ 不锈钢表面上形成的针化 膜在静态和旋转速度低于 $2.98 \mathrm{~m} \cdot \mathrm{s}^{-1}$ 时较稳定, 但 其点蚀电位明显低于复合层 $\mathrm{A}$ 和单一合金层. 旋转 速度增加到 $3.45 \mathrm{~m} \cdot \mathrm{s}^{-1}$ 时, 复合层 $\mathrm{B}$ 和 $316 \mathrm{~L}$ 不锈钢 没有观察到明显的钝化, 证实高转速条件下两者表 面钝化膜修复能力较差, 表面钝化膜已遭到破坏. 值 得注意的是, 静态条件下, 复合层 $\mathrm{A}$ 的 $i_{\mathrm{corr}}$ 大于单一 合金层的 $i_{\text {corr }}$; 而动态冲蚀条件下, 复合层 $\mathrm{A}$ 的 $i_{\text {corr }}$ 小于单一合金层的 $i_{\text {corr }}$

\section{4 交流阻抗测试}

图 5 给出了 316L 不锈钢、单一合金层、复合层
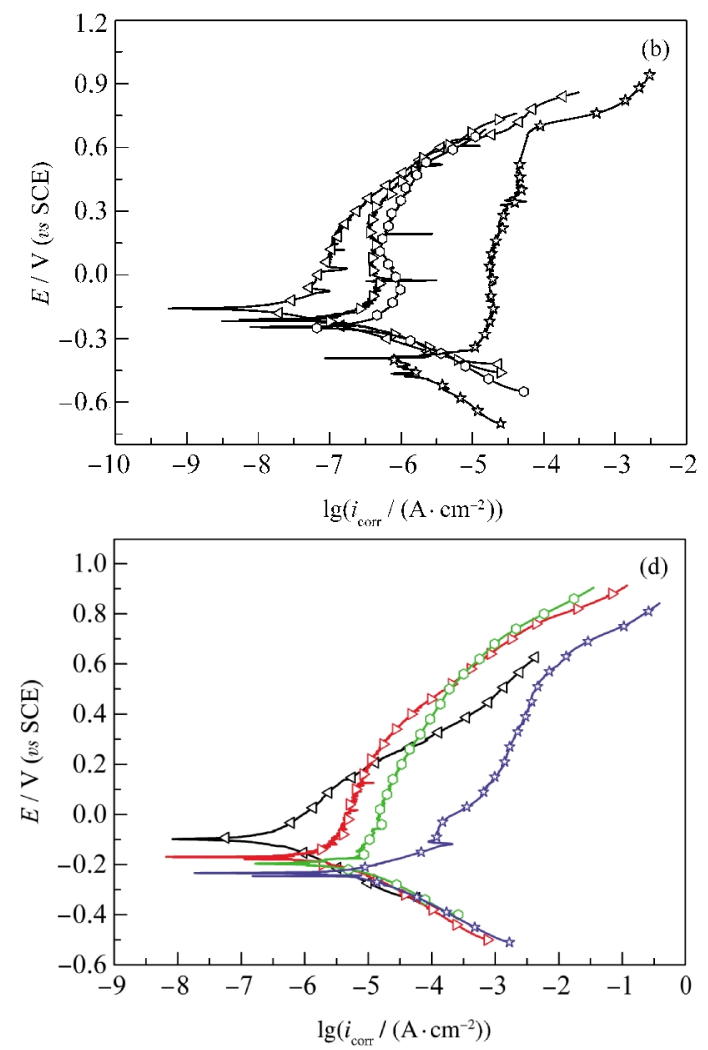

图 $4316 \mathrm{~L}$ 不锈钢基材 $(\mathrm{a})$ 、单一合金层 $(\mathrm{b})$ 、复合层 $\mathrm{A}(\mathrm{c})$ 和复合层 $\mathrm{B}(\mathrm{d})$ 在料浆流 $(3.5 \% \mathrm{NaCl}+10 \%$ 石英砂)中不同旋转速度 冲蚀条件下的极化曲线

Fig.4 Polarization curves of 316L stainless steel (a), single Ni-based alloying layer (b), composite layer A (c) and layer $\mathrm{B}(\mathrm{d})$ obtained in slurry flow $(3.5 \% \mathrm{NaCl}$ solution $+10 \%$ sand particles) with different rotational speeds 
$\mathrm{A}$ 和复合层 $\mathrm{B}$ 分别在 $3.5 \% \mathrm{NaCl}$ 溶液中浸泡 $20 \mathrm{~h}$ 、 在单相流 $(3.5 \% \mathrm{NaCl}$ 溶液)和在料浆流 $(3.5 \% \mathrm{NaCl}+$ $10 \%$ 石英砂)中冲蚀 $20 \mathrm{~h}$ 后, 开路电位下的交流阻抗 谱图. 由图可见, 几种测试材料的阻抗谱均呈现容抗 弧特征. 对比浸泡 $20 \mathrm{~h}$ 后的阻抗谱, 随着旋转速度 的增加以及石英砂的加人, 容抗弧幅值逐渐减小. 对 比复合层 $\mathrm{A}$ 、复合层 $\mathrm{B}$ 和单一合金层: 浸泡 $20 \mathrm{~h}$ 后, 容抗弧的大小顺序为单一合金层 $>$ 复合层 $\mathrm{A}>$ 复合 层 $\mathrm{B}$; 单相流和料浆流冲蚀 $20 \mathrm{~h}$ 后, 容抗弧的大小 顺序为复合层 $\mathrm{A}>$ 单一合金层 $>$ 复合层 $\mathrm{B}$. 由此可见: 静态条件下, 复合层 $\mathrm{A}$ 和复合层 $\mathrm{B}$ 的耐蚀性能均低 于单一合金层的耐蚀性能; 经过单相流和料浆流 20 $\mathrm{h}$ 的冲蚀后, 单一合金层的耐蚀性能低于复合层 $\mathrm{A}$, 但明显优于复合层 B. 在三种测试条件下, 316L 不 锈钢的容抗弧幅值最小, 其耐蚀性能最差.

\section{5 冲蚀截面形貌及冲蚀机理探讨}

图 6 为 316L 不锈钢、单一合金层、复合层 $\mathrm{A}$ 和 复合层 $\mathrm{B}$ 在料浆 $(3.5 \% \mathrm{NaCl}+10 \%$ 石英砂 $)$ 中, 旋转速 度为 $3.45 \mathrm{~m} \cdot \mathrm{s}^{-1}$ 条件下, 冲蚀 $20 \mathrm{~h}$ 后的截面形貌
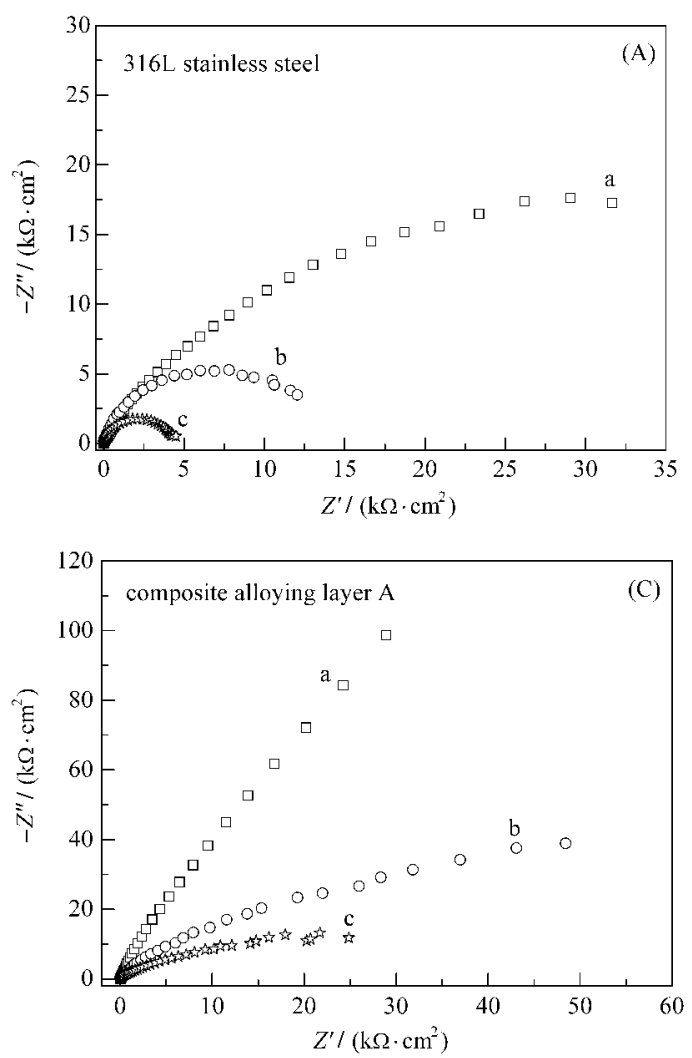

SEM 照片. 由图 6(a)所示, 316L 不锈钢表层出现裂 纹和脆性剥落; 同时可观察到裂纹沿垂直和平行表 层方向扩展. 在硬质颗粒切削下, 导致金属表面保护 膜的破裂和脱落, 暴露出的“新鲜”基体加速了材料 的腐蚀; 此外高速液固双相流的冲击, 材料表面产 生大量形变, 形成“不同应变区原电池”, 加速了材料 的流失 ${ }^{[23]}$.

单一合金层的截面形貌(图 6(b))较平整, 没有 裂纹和点蚀孔的形成, 呈现出典型的均匀腐蚀特征. 极化曲线、交流阻抗谱以及外加电位条件下的电流 密度测试都表明单一合金层具有较好的耐蚀性. 但 是在冲蚀条件下, 钝化膜的去除和修复是动态平衡 过程. 由于单一合金层硬度较低 $\left(202 \mathrm{HV}_{0.1}\right)$, 在硬颗 粒冲击合金表面时, 易于去除表层钝化膜. 在低转速 条件下, 钝化膜还具有良好的修复能力; 而在高转 速条件下, 料浆介质中的硬颗粒对钝化膜的去除能 力增强, 导致其耐冲蚀性能下降. 因此, 针化膜的修 复能力和稳定性是控制单一合金层冲蚀性能的主要 因素.
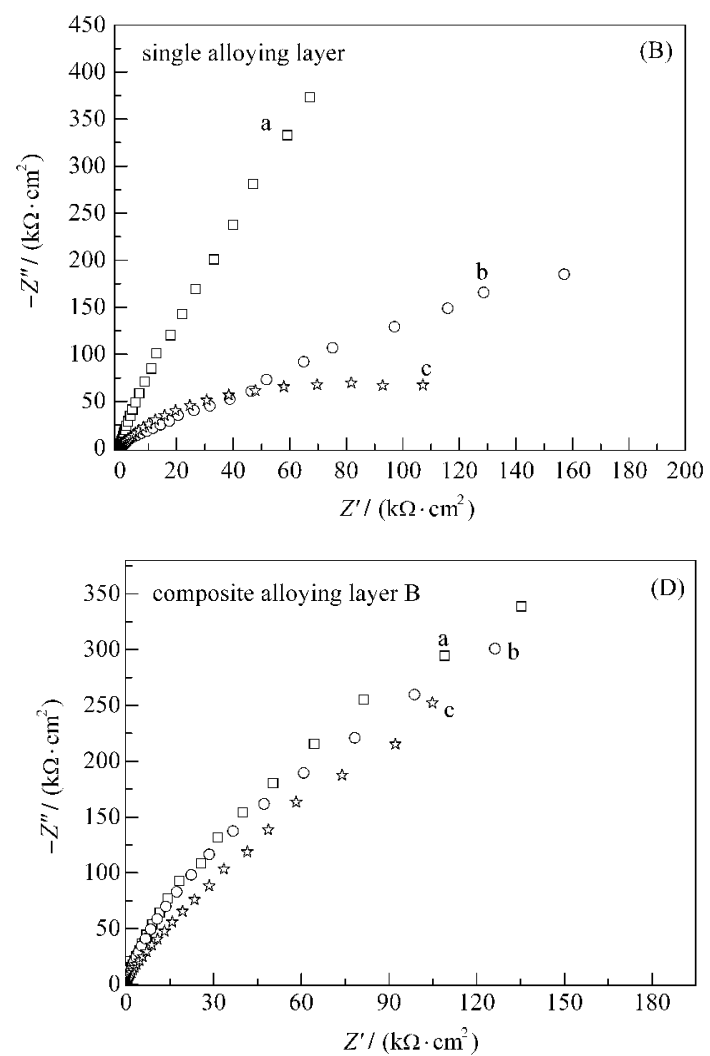

图 5 四种测试材料分别在静态浸泡(3.5\% NaCl 溶液) $20 \mathrm{~h}$ (a)以及在单相流(3.5\% $\mathrm{NaCl}$ 溶液)(b)和料浆流( $3.5 \% \mathrm{NaCl}+$ $10 \%$ 石英砂)(c)条件下冲蚀 $20 \mathrm{~h}$ 后阻抗谱 Nyquist 图

Fig.5 Nyquist plots of impedance spectra for samples after immersion in $3.5 \% \mathrm{NaCl}$ solution for $20 \mathrm{~h}$ (a), erosion-corrosion test in liquid flow $(3.5 \% \mathrm{NaCl}$ solution) $(\mathrm{b})$, and in slurry flow

$(3.5 \% \mathrm{NaCl}$ solution $+10 \%$ sand particles) (c) for $20 \mathrm{~h}$ 

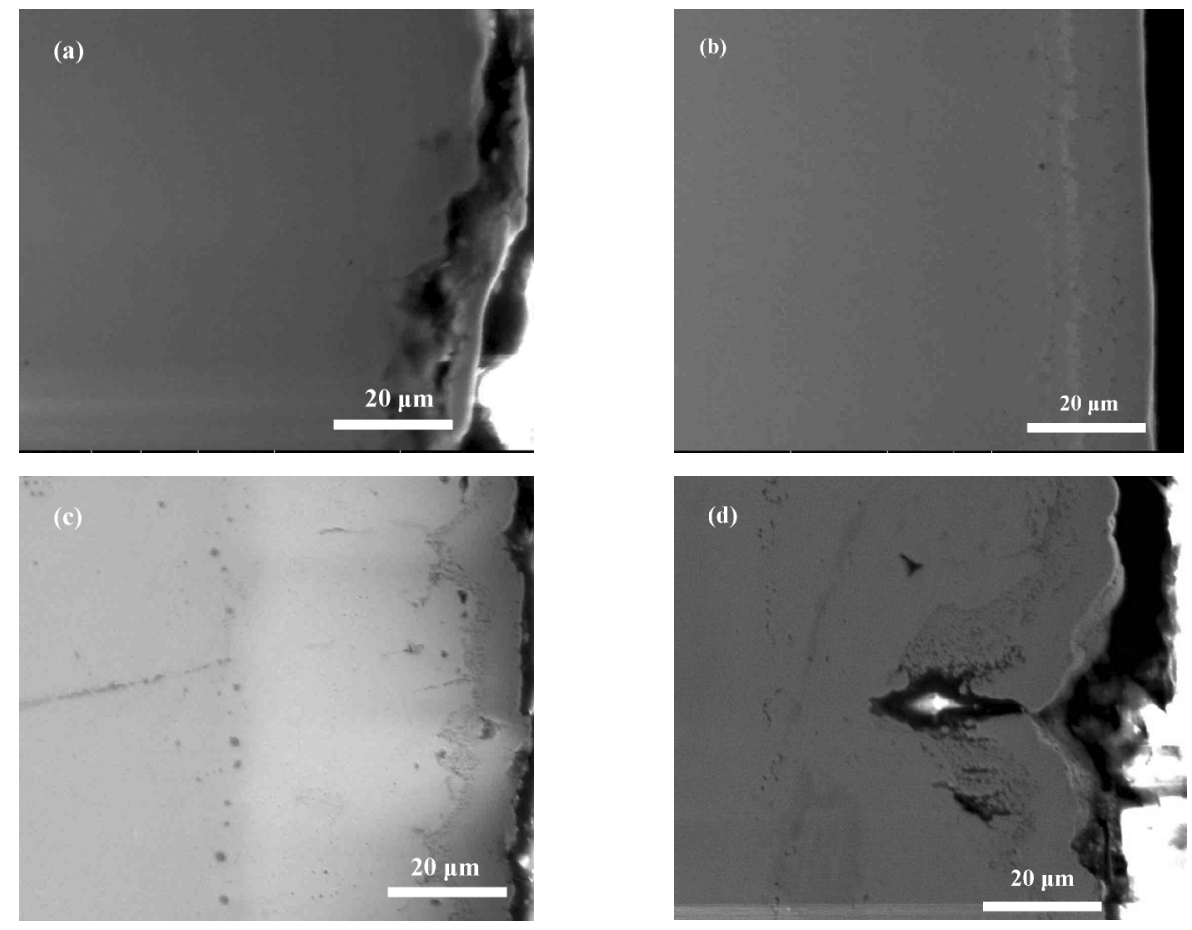

图 $6316 \mathrm{~L}$ 不锈钢 $(\mathrm{a})$ 、单一合金层 $(\mathrm{b})$ 、复合层 $\mathrm{A}(\mathrm{c})$ 和复合层 $\mathrm{B}(\mathrm{d})$ 在料浆流中冲蚀 $20 \mathrm{~h}$ 后的腐蚀截面形貌

Fig.6 SEM images of the cross-section surface morphologies of $316 \mathrm{~L}$ stainless steel (a), single Ni-based alloying layer (b), composite layer A (c) and composite layer B (d ) after $20 \mathrm{~h}$ of erosion-corrosion test in slurry flow rotation velocity: $3.45 \mathrm{~m} \cdot \mathrm{s}^{-1}$

与单一合金层相似, 复合层 $\mathrm{A}$ 的截面冲蚀形貌 (图 6(c)) 较平整, 可见添加的纳米 $\mathrm{SiO}_{2}$ 颗粒实现了 复合层 $\mathrm{A}$ 具有优良的耐蚀性和耐磨性的最佳配合. 纳米 $\mathrm{SiO}_{2}$ 颗粒对复合层耐冲蚀性能的提高主要有三 个方面的作用: (1) 纳米 $\mathrm{SiO}_{2}$ 颗粒与 $\mathrm{Ni}$ 基体结合紧 密, 界面干净无孔洞, 这样既实现了 $\mathrm{Ni}$ 基体对颗粒提 供有效的支撑, 同时有效组织 $\mathrm{Cl}^{-}$在相界面发生腐蚀; (2) 纳米 $\mathrm{SiO}_{2}$ 颗粒本身的电阻率为 $1 \times 10^{14} \Omega \cdot \mathrm{cm}^{2}{ }^{[24]}$, 为非导体相, 具有屏蔽效应, 减少了合金基体与腐蚀 介质的接触面积, 从而有效提高耐蚀性能; (3) 纳米 $\mathrm{SiO}_{2}$ 颗粒均匀弥散分布在 $\mathrm{Ni}$ 基体内, 由于纳米 $\mathrm{SiO}_{2}$ 颗粒对位错运动的阻碍以及增殖作用, 显著改 善了复合层 $\mathrm{A}$ 的弥散强化能力, 与单一合金层相比, 有效地提高复合层 $\mathrm{A}$ 的硬度 $\left(368 \mathrm{HV}_{0.1}\right)$; 减轻了腐蚀 介质中硬颗粒对 $\mathrm{Ni}$ 基体的犁削破坏作用, 有效地提 高了钝化膜的稳定性. 因此, 复合层 $\mathrm{A}$ 具有最优的 耐冲蚀性能.

对于复合层 $\mathrm{B}$ (图 6(d)), 冲蚀后表层凹凸不平, 并伴有尺寸较大的的点蚀坑存在. 尽管复合层 $\mathrm{B}$ 的 硬度较单一合金层有了提高, 能有效抵抗腐蚀介质 中硬颗粒的犁削破坏, 但存在的第二相对复合层 B 的腐蚀性能不利. 由于添加的纳米 $\mathrm{SiC}$ 颗粒完全分
解析出 $\mathrm{Cr}_{23} \mathrm{C}_{6}$ 和 $\mathrm{Cr}_{6.5} \mathrm{Ni}_{2.5} \mathrm{Si}$, 造成在析出相周围产生 贫 $\mathrm{Cr}$ 区, 贫 $\mathrm{Cr}$ 区电极电位较低, 易于发生优先活性 溶解, 成为活性状态的阳极, 而周围基体保持原有的 合金含量, 电位较高处于钝态, 作为阴极, 从而构成 大阴极(基体)-小阳极(贫 $\mathrm{Cr}$ 区)的腐蚀微电池, 降低 了复合层 $\mathrm{B}$ 的耐蚀性能. 在动态冲蚀条件下, 料浆 流加速传质过程, 使得复合层 $\mathrm{B}$ 活性溶解趋势增强; 同时由于析出相周围优先发生腐蚀, 导致析出相在 悬浮颗粒冲击作用下易于脱落, 进而加速复合层 B 的质量流失.

\section{3 结 论}

(1) 纳米颗粒增强 $\mathrm{Ni}$ 基复合镀渗层中添加的纳 米 $\mathrm{SiO}_{2}$ 颗粒仍保持非晶态; 而纳米 $\mathrm{SiC}$ 颗粒已完全 分解并与 $\mathrm{Ni} 、 \mathrm{Cr}$ 反应析出三元硅化物 $\mathrm{Cr}_{6.5} \mathrm{Ni}_{2.5} \mathrm{Si}$ 和 碳化物 $\mathrm{Cr}_{23} \mathrm{C}_{6}$.

(2) 外加电位 $(+0.2 \mathrm{~V})$ 条件下的电流密度测试、 动态极化曲线和三种条件下的交流阻抗结果表明: 静态条件下, 电刷镀含纳米 $\mathrm{SiO}_{2}$ 颗粒的复合镀渗层 的耐蚀性能略低于单一合金层, 而动态冲蚀条件下, 电刷镀含 $\mathrm{SiO}_{2}$ 颗粒增强的复合镀渗层的耐蚀性能 则优于单一合金层; 电刷镀含 $\mathrm{SiC}$ 颗粒增强的复合 
镀渗层的耐蚀性能始终低于单一合金层.

(3) 由于弥散分布的纳米 $\mathrm{SiO}_{2}$ 颗粒对位错运动 的阻碍以及增殖作用, 提高了复合层 $\mathrm{A}$ 的硬度, 有 效地阻挡砂粒对钝化膜的犁削作用, 提高了 $\mathrm{Ni}$ 基合 金层的耐冲蚀性能; 而对于复合层 $\mathrm{B}$, 由于添加的纳 米 $\mathrm{SiC}$ 颗粒已完全分解, 并析出了三元硅化物和碳 化物, 析出相明显降低了 $\mathrm{Ni}$ 基合金层的耐蚀性能和 耐冲蚀性能.

\section{References}

1 Zhang, G. A.; Cheng, Y. F. Corrosion Sci., 2009, doi: 10.1016/j. corsci.2009.01.020

2 Matthews, S.; James, B.; Hyland, M. Surf. Coat. Technol., 2009, 203: 1086

3 Wood, R. J. K.; Speyer, A. J. Wear, 2004, 256: 545

4 Zhang, G. A.; Xu, L. Y.; Cheng, Y. F. Corrosion Sci., 2009, 51: 283

5 Zhang, A. F.; Wang, Y. Y.; Xing, J. D.; Li, C. J. Acta Metall. Sin., 2004, 40: 411 [张安峰, 王豫跃, 邢建东, 李长久. 金属学报, 2004, 40: 411]

6 Szczygieł, B.; Kołodziej, M. Electrochim. Acta, 2005, 50: 4188

7 Pardo, A.; Merino, S.; Merino, M. C.; Barroso, I.; Mohedano, M.; Arrabal, R.; Viejo, F. Corrosion Sci., 2009, 51: 814

8 Singh, I. B.; Mandal, D. P.; Singh, M.; Das, S. Corrosion Sci., 2009, 51: 234
9 Espallargas, N.; Berget, J.; Guilemany, J. M.; Benedetti, A. V.; Suegama, P. H. Surf. Coat. Technol., 2008, 202: 1405

10 Tu, J. P.; Li, C. L.; Zhao, H. X.; Matsumura, M. Mater. Corros., 1999, 50: 205

11 Das, S.; Saraswathi, Y. L.; Mondal, D. P. Wear, 2006, 261: 180

12 Das, S.; Mondal, D. P.; Dasgupta, R. Wear, 1999, 231: 195

13 Burstein, G. T.; Sasaki, K. Electrochim. Acta, 2001, 46: 3675

14 Burstein, G. T.; Sasaki, K. Wear, 2000, 240: 80

15 Smith, A. J.; Stratmann, M.; Hassel, A. W. Electrochim. Acta, 2006, 51: 6521

16 Akiyama, E.; Stratmann, M.; Hassel, A. W. J. Phys. D-Appl. Phys., 2006, 39: 3157

17 Yu, H.; Zheng, Y. G.; Yao, Z. M. Mater. Corros., 2006, 57: 705

18 Tang, X.; Xu, L. Y.; Cheng, Y. F. Corrosion Sci., 2008, 50: 1469

19 Xu, J.; Xie, X. S. Acta Metall. Sin., 2002, 38: 1074 [徐 江, 谢锡善. 金属学报, 2002, 38: 1074]

20 Jiang, X.; Cheng, Z. Z.; Jie, T.; Shu, Y. J.; Lin, L. L. J. Phys. DAppl. Phys., 2009, 42: 3

21 Zheng, Y. G.; Yao, Z. M.; Wei, X. Y.; Ke, W. Wear, 1995, 186187: 555

22 Ambat, R.; Davenport, A, J.; Scamans, G. M.; Afseth, A. Corrosion Sci., 2006, 48: 3455

23 Heidemeyer, J. Wear, 1981, 66: 379

24 Chen, X.; Han, M. F.; Wang, Z. L.; Jiang, X. F. Rare Met. Mater. Eng., 2007, 36: 642 [陈 釒金, 韩敏芳, 王忠利, 蒋先锋. 稀有金 属材料与工程, 2007, 36: 642] 